Pacific Journal of Mathematics

PAIRS OF POSITIVE SOLUTIONS OF QUASILINEAR
ELLIPTIC EQUATIONS IN EXTERIOR DOMAINS

T es andrew Swanson and Hiroyuki Us ami 


\section{PAIRS OF POSITIVE SOLUTIONS OF QUASILINEAR ELLIPTIC EQUATIONS IN EXTERIOR DOMAINS}

\section{Takasi Kusano, Charles A. Swanson and Hiroyuki Usami}

Our main objective is to prove the existence of infinitely many pairs $\left(u_{1}, u_{2}\right)$ of positive solutions of quasilinear elliptic differential equations

$$
\Delta u-q(|x|) u=f(x, u, \nabla u), \quad x \in \Omega_{\alpha},
$$

throughout exterior domains $\Omega_{\alpha} \subset \mathbf{R}^{N}, N \geq 2$, of the type

$$
\Omega_{\alpha}=\left\{x \in \mathbf{R}^{N}:|x|>\alpha\right\}, \quad \alpha>0,
$$

where $x=\left(x_{1}, \ldots, x_{N}\right), \nabla u=\left(\partial u / \partial x_{1}, \ldots, \partial u / \partial x_{N}\right)$, and $\Delta=\nabla \cdot \nabla$. Each pair has the property that $u_{1}(x) / u_{2}(x)$ has uniform limit zero in $\Omega_{\alpha}$ as $|x| \rightarrow \infty$. In particular, if $q(t) \equiv 0$ and $N \geq 3, u_{1}(x)$ has limit 0 as $|x| \rightarrow \infty$, and $u_{2}(x)$ is bounded above and below by positive constants in $\Omega_{\alpha}$.

1. The function $q$ in (1.1) is assumed to be nonnegative and locally Hölder continuous in $\mathbf{R}_{+}=[0, \infty)$, and $f: \Omega_{\alpha} \times \mathbf{R}_{+} \times \mathbf{R}^{N} \rightarrow \mathbf{R}$ is locally Hölder continuous in $\Omega_{\alpha} \times \mathbf{R}_{+} \times \mathbf{R}^{N}$ and satisfies a Nagumo condition. Detailed hypotheses are listed in $\S 3$.

Specific asymptotic estimates for the growth (decay) of the solutions $u_{1}(x), u_{2}(x)$ as $|x| \rightarrow \infty$ follow easily from our construction. In particular sufficient conditions are given for the quasilinear equation

$$
\Delta u-|x|^{2 r} u=\phi(x) u^{\gamma}+\psi(x)|\nabla u|^{\beta}, \quad x \in \Omega_{\alpha},
$$

for constants $r \geq 0, \gamma \geq 0,0 \leq \beta \leq 2$, to have positive solutions $u_{1}(x)$, $u_{2}(x)$ in $\Omega_{\alpha}$ such that $u_{i}(x)$ is bounded above and below by positive constant multiples of

$$
|x|^{-\lambda} \exp \left[(-1)^{l}|x|^{r+1} /(r+1)\right], \quad x \in \Omega_{\alpha}, i=1,2,
$$

where $\lambda=(N+r-1) / 2$.

By a similar method we also prove the existence of infinitely many positive solutions of the boundary value problem

$$
\begin{aligned}
& \Delta u-q(|x|) u=f(x, u, \nabla u), \quad x \in \Omega_{\alpha}, \\
& \left.u\right|_{\partial \Omega_{\alpha}}=0
\end{aligned}
$$

under the same hypotheses as for (1.1). 
The sharpness of our results is indicated by known oscillation criteria for semilinear elliptic equations [6, 10]. For example, in the case $\psi(x)=0$ in equation (3.26), our conditions (3.27)-(3.30) are all known to be necessary and sufficient conditions for the existence of a positive solution of (3.26), of the type described in Corollaries 3.8-3.11, in some exterior domain [11].

Our procedure will be to construct solutions of (1.1) or (1.2) which are squeezed between subsolutions and supersolutions. The latter are obtained as spherically symmetric solutions of elliptic equations with $f(x, u, \nabla u)$ in (1.1) replaced by radial majorants. We therefore begin by proving global existence theorems for quasilinear ordinary differential equations (2.1) below. These results have independent interest, and in fact are of quite general nature. The procedure will be to find solutions of integro-differential equations as fixed points of associated operators from closed convex subsets $\mathscr{Y}$ of $C^{1}\left[t_{0}, \infty\right)$ into subsets of $\mathscr{Y}$ with compact closure.

In the case of semilinear equations $\Delta u=f(x, u)$, in which $q(t) \equiv 0$, global existence theorems for boundary value problems in exterior domains have been obtained by Kawano and Naito [2], Kenig and Ni [3], and Noussair and Swanson [8]. Existence of positive bounded solutions in the entire space $\mathbf{R}^{N}, N \geq 3$, have been proved by Kawano [1], Kusano and Oharu [4], and $\mathrm{Ni}$ [5].

2. Positive solutions of quasilinear ordinary differential equations. Existence theorems will be proved for quasilinear ordinary differential equations of the type

$$
L y=h\left(t, y, y^{\prime}\right), \quad t \geq t_{0}>0,
$$

where $L$ is the linear differential operator defined by

$$
L z=\frac{1}{p_{2}(t)} \frac{d}{d t}\left[\frac{1}{p_{1}(t)} \frac{d}{d t}\left(\frac{z}{p_{0}(t)}\right)\right], \quad z \in C^{2}\left[t_{0}, \infty\right) .
$$

AsSUMPTIONS.

$\left(\mathrm{A}_{1}\right)$ Each $p_{i}(t)$ is positive in $\left[t_{0}, \infty\right), p_{i} \in C^{2-i}\left[t_{0}, \infty\right), i=0,1,2$, and $\lim _{t \rightarrow \infty} P(t)=+\infty$, where

$$
P(t)=\int_{t_{0}}^{t} p_{1}(s) d s .
$$
fies

$\left(\mathrm{A}_{2}\right) h:\left[t_{0}, \infty\right) \times \mathbf{R}_{+} \times \mathbf{R} \rightarrow \mathbf{R}, \mathbf{R}_{+}=[0, \infty)$, is continuous and satis-

$$
|h(t, y, z)| \leq H(t,|y|,|z|)
$$


for all $t \in\left[t_{0}, \infty\right), y \in \mathbf{R}_{+}, z \in \mathbf{R}$, where $H(t, u, v)$ is continuous in $\left[t_{0}, \infty\right) \times \mathbf{R}_{+} \times \mathbf{R}_{+}$, nondecreasing in $u$ for each $t, v$, and nondecreasing in $v$ for each $t, u$.

The notation below will be used throughout:

$$
p(t)=p_{0}(t) p_{1}(t) / P(t)+\left|p_{0}^{\prime}(t)\right| .
$$

For $t>t_{0},\left(\mathrm{~A}_{1}\right),\left(\mathrm{A}_{2}\right)$ and (2.2) show that the linear equation $L z=0$ has linearly independent positive solutions

$$
z_{1}(t)=p_{0}(t), \quad z_{2}(t)=p_{0}(t) P(t),
$$

which are asymptotically ordered, i.e., $\lim _{t \rightarrow \infty} z_{1}(t) / z_{2}(t)=0$. The objective of this section is to establish sufficient conditions for equation (2.1) to have solutions $y_{1}(t)$ and $y_{2}(t)$ on the entire interval $\left[t_{0}, \infty\right)$ with the same asymptotic behavior as $z_{1}(t)$ and $z_{2}(t)$, respectively, as $t \rightarrow \infty$. We also prove the existence of a positive solution $y(t)$ of $(2.1)$ in $\left(t_{0}, \infty\right)$ satisfying the boundary condition $y\left(t_{0}\right)=0$.

It is well known [12] that every nonoscillatory linear differential operator $L$ of second order in $(0, \infty)$ can be written in the factorized form (2.2), where

$$
p_{0}=z_{1}, \quad p_{1}=\left(z_{2} / z_{1}\right)^{\prime}, \quad p_{2}=1 / p_{0} p_{1}
$$

for linearly independent, asymptotically ordered solutions $z_{1}(t)$ and $z_{2}(t)$ of $L z=0$, provided $t_{0}$ is large enough so that these solutions are positive in $\left[t_{0}, \infty\right)$. The functions (2.4) are unique up to multiplicative constants.

The following hypotheses are used in the theorem below:

$$
\begin{gathered}
\int_{t_{0}}^{\infty} p_{2}(t) P(t) H\left(t, a p_{0}(t), b p(t)\right) d t<\infty ; \\
\int_{t_{0}}^{\infty} p_{2}(t) H\left(t, a p_{0}(t) P(t), b p(t) P(t)\right) d t<\infty
\end{gathered}
$$

for some positive constants $a$ and $b$.

THEOREM 2.1. In addition to $\left(\mathrm{A}_{1}\right),\left(\mathrm{A}_{2}\right)$ suppose that $\lambda^{-1} H(t, \lambda u, \lambda v)$ is a nondecreasing function of $\lambda \in(0, \infty)$ and

$$
\lim _{\lambda \rightarrow 0+} \lambda^{-1} H(t, \lambda u, \lambda v)=0
$$

for each fixed $(t, u, v) \in\left[t_{0}, \infty\right) \times \mathbf{R}_{+} \times \mathbf{R}_{+}$.

(i) Condition (2.5) implies that equation (2.1) has infinitely many positive (negative) solutions $y(t)$ in $\left[t_{0}, \infty\right)$ such that $\lim _{t \rightarrow \infty} y(t) / z_{1}(t)$ exists and is positive (negative, respectively). 
(ii) Condition (2.6) implies that equation (2.1) has infinitely many positive (negative) solutions $y(t)$ in $\left(t_{0}, \infty\right)$ such that $\lim _{t \rightarrow \infty} y(t) / z_{2}(t)$ exists and is positive (negative, respectively).

Proof of (i). Let $m=\min \{a, b\}$. The nondecreasing hypothesis on $\lambda^{-1} H(t, \lambda u, \lambda v)$ shows that

$$
\begin{aligned}
\lambda^{-1} p_{2}(t) P(t) & H\left(t, \lambda p_{0}(t), \lambda p(t)\right) \\
\leq & m^{-1} p_{2}(t) P(t) H\left(t, m p_{0}(t), m p(t)\right)
\end{aligned}
$$

for $0<\lambda \leq m$ and for all $t \in\left[t_{0}, \infty\right)$. By $\left(\mathrm{A}_{2}\right)$ and (2.5), the right member of (2.8) is integrable in $\left(t_{0}, \infty\right)$, and by (2.7) the left member has limit zero as $\lambda \rightarrow 0+$ at every $t \in\left[t_{0}, \infty\right)$. The dominated convergence theorem then implies that

$$
\lim _{\lambda \rightarrow 0+} \int_{t_{0}}^{\infty} \lambda^{-1} p_{2}(t) P(t) H\left(t, \lambda p_{0}(t), \lambda p(t)\right) d t=0,
$$

and therefore a sufficiently small constant $k>0$ exists such that

$$
\int_{t_{0}}^{\infty} p_{2}(t) P(t) H\left(t, 3 k p_{0}(t), 3 k p(t)\right) d t \leq k .
$$

With this choice of $k$ we consider

$$
\begin{aligned}
& \mathscr{Y}=\left\{y \in C^{1}\left[t_{0}, \infty\right): k p_{0}(t) \leq y(t) \leq 3 k p_{0}(t),\right. \\
&\left.\left|y^{\prime}(t)\right| \leq 3 k p(t), t \geq t_{0}\right\},
\end{aligned}
$$

where $C^{1}\left[t_{0}, \infty\right)$ denotes the locally convex space of all continuously differentiable functions in $\left[t_{0}, \infty\right)$ with the topology of uniform convergence of functions and their first derivatives on compact subintervals of $\left[t_{0}, \infty\right)$. It is clear that $\mathscr{Y}$ is a closed convex subset of $C^{1}\left[t_{0}, \infty\right)$. Define the integro-differential operator $M$ by

(2.11) $(M y)(t)$

$$
=p_{0}(t)\left[2 k+\int_{t}^{\infty}\left(\int_{t}^{s} p_{1}(\sigma) d \sigma\right) p_{2}(s) h\left(s, y(s), y^{\prime}(s)\right) d s\right],
$$

If $y \in \mathscr{Y},(2.9)-(2.11)$ show that

$$
\left|\frac{(M y)(t)}{p_{0}(t)}-2 k\right| \leq \int_{t_{0}}^{\infty} p_{2}(s) P(s) H\left(s, 3 k p_{0}(s), 3 k p(s)\right) d s \leq k
$$


and

$$
\begin{gathered}
\left|(M y)^{\prime}(t)\right| \leq\left|p_{0}^{\prime}(t)\right|\left[2 k+\int_{t_{0}}^{\infty} p_{2}(s) P(s) H\left(s, 3 k p_{0}(s), 3 k p(s)\right) d s\right] \\
\quad+p_{0}(t) p_{1}(t) \int_{t}^{\infty} p_{2}(s) H\left(s, 3 k p_{0}(s), 3 k p(s)\right) d s \\
\leq 3 k\left|p_{0}^{\prime}(t)\right|+\frac{p_{0}(t) p_{1}(t)}{P(t)} \int_{t}^{\infty} p_{2}(s) P(s) H\left(s, 3 k p_{0}(s), 3 k p(s)\right) d s \\
\leq 3 k\left|p_{0}^{\prime}(t)\right|+k p_{0}(t) p_{1}(t) / P(t)<3 k p(t) .
\end{gathered}
$$

Therefore $M$ maps $\mathscr{Y}$ into $\mathscr{Y}$. The continuity of $M$ follows from (2.9) and the dominated convergence theorem. The fact that $M \mathscr{Y}$ has compact closure can be easily checked via Ascoli's theorem. It then follows from the Schauder-Tychonoff fixed point theorem that $M$ has a fixed point $y \in \mathscr{Y}$. Differentiation of the equation $y=M y$ twice shows that $y(t)$ is a positive solution of $(2.1)$ in $\left[t_{0}, \infty\right)$, and clearly $\lim _{t \rightarrow \infty} y(t) / p_{0}(t)=2 k$ by (2.11). Since infinitely many distinct choices of $k$ are possible in (2.9), equation (2.1) has infinitely many positive solutions $y(t)$ such that $\lim _{t \rightarrow \infty} y(t) / p_{0}(t)$ exists and is positive. A slight modification establishes the analogous statement for negative solutions.

Proof of (ii). It follows as in part (i) that

$$
\lim _{\lambda \rightarrow 0+} \int_{t_{0}}^{\infty} \lambda^{-1} p_{2}(t) H\left(t, \lambda p_{0}(t) P(t), \lambda p(t) P(t)\right) d t=0,
$$

from which a sufficiently small constant $k>0$ exists such that

$$
\int_{t_{0}}^{\infty} p_{2}(t) H\left(t, 3 k p_{0}(t) P(t), 3 k p(t) P(t)\right) d t \leq k .
$$

With $k$ as in (2.12), we define analogues $\tilde{\mathscr{Y}}$ and $\tilde{M}$ of (2.10) and (2.11), respectively, by

$$
\begin{aligned}
\tilde{\mathscr{Y}}=\left\{y \in C^{1}\left[t_{0}, \infty\right): k p_{0}(t) P(t) \leq y(t)\right. & \leq 3 k p_{0}(t) P(t), \\
\left|y^{\prime}(t)\right| & \left.\leq 3 k p(t) P(t), t \geq t_{0}\right\},
\end{aligned}
$$

(2.14) $(\tilde{M} y)(t)$

$$
=p_{0}(t)\left[2 k P(t)-\int_{t_{0}}^{t} p_{1}(s) \int_{s}^{\infty} p_{2}(\sigma) h\left(\sigma, y(\sigma), y^{\prime}(\sigma)\right) d \sigma d s\right],
$$

$$
t \geq t_{0} \text {. }
$$


If $y \in \tilde{\mathscr{Y}}$, one finds from (2.12) and (2.14) upon interchanging the order of integration that

$$
\begin{aligned}
& \left|\frac{(\tilde{M} y)(t)}{p_{0}(t)}-2 k P(t)\right| \\
& \quad \leq P(t) \int_{t_{0}}^{\infty} p_{2}(\sigma) H\left(\sigma, 3 k p_{0}(\sigma) P(\sigma), 3 k p(\sigma) P(\sigma)\right) d \sigma \\
& \quad \leq k P(t),
\end{aligned}
$$

and

$$
\begin{aligned}
& \left|(\tilde{M} y)^{\prime}(t)\right| \\
& \leq\left|p_{0}^{\prime}(t)\right|\left[2 k P(t)+P(t) \int_{t_{0}}^{\infty} p_{2}(\sigma) H\left(\sigma, 3 k p_{0}(\sigma) P(\sigma), 3 k p(\sigma) P(\sigma)\right) d \sigma\right] \\
& \quad+p_{0}(t) p_{1}(t)\left[2 k+\int_{t_{0}}^{\infty} p_{2}(\sigma) H\left(\sigma, 3 k p_{0}(\sigma) P(\sigma), 3 k p(\sigma) P(\sigma)\right) d \sigma\right] \\
& \leq 3 k\left|p_{0}^{\prime}(t)\right| P(t)+3 k p_{0}(t) p_{1}(t)=3 k p(t) P(t), \quad t \geq t_{0} .
\end{aligned}
$$

This shows that $\tilde{M}$ maps $\tilde{\mathscr{Y}}$ into $\tilde{\mathscr{Y}}$. The remainder of the proof closely parallels that of part (i) and will be deleted.

THEOREM 2.2. In addition to $\left(\mathrm{A}_{1}\right)$ and $\left(\mathrm{A}_{2}\right)$ suppose that $\mu^{-1} H(t, \mu u, \mu v)$ is a nonincreasing function of $\mu \in(0, \infty)$ and

$$
\lim _{\mu \rightarrow \infty} \mu^{-1} H(t, \mu u, \mu v)=0
$$

for each fixed $(t, u, v) \in\left[t_{0}, \infty\right) \times \mathbf{R}_{+} \times \mathbf{R}_{+}$. Then both conclusions (i) and (ii) of Theorem 2.1 hold verbatim et literatim.

Proof. Let $C=\max \{a, b\}$. Then, in analogy with (2.8),

$$
\mu^{-1} p_{2}(t) P(t) H\left(t, \mu p_{0}(t), \mu p(t)\right) \leq C^{-1} p_{2}(t) P(t) H\left(t, C p_{0}(t), C p(t)\right)
$$

for all $\mu \geq C$ and $t \in\left[t_{0}, \infty\right)$. It follows from $\left(\mathrm{A}_{2}\right),(2.5)$ and (2.15) exactly as in Theorem 2.1 that

$$
\lim _{\mu \rightarrow \infty} \int_{t_{0}}^{\infty} \mu^{-1} p_{2}(t) P(t) H\left(t, \mu p_{0}(t), \mu p(t)\right) d t=0,
$$

and hence there exists a sufficiently large positive constant $k$ such that (2.9) is satisfied. Proceeding exactly as in the proof of part (i) of Theorem 2.1, we obtain a positive solution $y(t)$ of $(2.1)$ in $\left[t_{0}, \infty\right)$ with $\lim _{t \rightarrow \infty} y(t) / p_{0}(t)=2 k>0$ as a fixed point of the operator $M$ in the set $\mathscr{Y}$. 
To prove part (ii), it suffices to observe that

$$
\lim _{\mu \rightarrow \infty} \mu^{-1} \int_{t_{0}}^{\infty} p_{2}(t) H\left(t, \mu p_{0}(t) P(t), \mu p(t) P(t)\right) d t=0 .
$$

This implies that (2.12) holds for sufficiently large positive constants $k$, and the proof is completed as in Theorem 2.1.

The proof of Theorem 2.1 (ii) also establishes the existence of infinitely many positive solutions of the quasilinear boundary value problem

$$
\begin{aligned}
& L y=h\left(t, y, y^{\prime}\right), \quad t \geq t_{0}, \\
& y\left(t_{0}\right)=0 .
\end{aligned}
$$

THeOREM 2.3. Suppose that $\left(\mathrm{A}_{1}\right)$ and $\left(\mathrm{A}_{2}\right)$ hold. Let $H$ be as in either Theorem 2.1 or Theorem 2.2. Then the condition (2.6) is sufficient for the boundary value problem (2.16) to have infinitely many positive solutions in $\left(t_{0}, \infty\right)$.

In fact, for infinitely many admissible constants $k$ in (2.6), Theorem 2.1 (ii) or Theorem 2.2 (ii) shows that equation (2.1) has a solution $y \in \tilde{\mathscr{Y}}$ satisfying $y(t)=(\tilde{M} y)(t)$ for $t \geq t_{0}$. Since $P\left(t_{0}\right)=0$ by $\left(\mathrm{A}_{1}\right), y(t)$ is a positive solution of $(2.16)$ in $\left(t_{0}, \infty\right)$ by (2.13) and (2.14).

3. Pairs of positive solutions of quasilinear elliptic equations. The radial component of the linear part of equation (1.1) (i.e., $f$ replaced by 0 ) is

$$
L z=t^{1-N} \frac{d}{d t}\left(t^{N-1} \frac{d z}{d t}\right)-q(t) z=0, \quad t \geq \alpha .
$$

Since (3.1) is nonoscillatory at $\infty$, there exist linearly independent positive solutions $z_{1}(t)$ and $z_{2}(t)$ of (3.1) on some fixed interval $\left[t_{0}, \infty\right)$, relabelled as $[\alpha, \infty)$, such that $\lim _{t \rightarrow \infty} z_{1}(t) / z_{2}(t)=0$. Then the operator $L$ has the factorized form (2.2) in $[\alpha, \infty)$, where $p_{0}, p_{1}$ and $p_{2}$ are defined by (2.4).

By applying the results of $\S 2$, we intend to find sufficient conditions for equation (1.1) to have positive solutions $u_{1}(x)$ and $u_{2}(x)$ in the entire domain $\Omega_{\alpha}$ with the same asymptotic behavior at $\infty$ as the functions $z_{1}(|x|)$ and $z_{2}(|x|)$, respectively. The standing hypotheses on equation (1.1) are listed below.

\section{ASSUMPTIONS.}

$\left(\mathrm{B}_{1}\right) q(t)$ is nonnegative and locally Hölder continuous in $\mathbf{R}_{+}$, and $f(x, u, p)$ is locally Hölder continuous in $\Omega_{\alpha} \times \mathbf{R}_{+} \times \mathbf{R}^{N}$. 
$\left(\mathrm{B}_{2}\right)$ For any bounded subdomain $G$ of $\Omega_{\alpha}$ and any constant $C>0$, there corresponds a constant $\rho(G, C)$ such that

$$
|f(x, u, p)| \leq \rho(G, C)\left(1+|p|^{2}\right)
$$

for all $x \in G, 0 \leq u \leq C$, and $p \in \mathbf{R}^{N}$ (Nagumo condition).

$\left(\mathrm{B}_{3}\right)$ There exists a continuous function $F: \mathbf{R}_{+}^{3} \rightarrow \mathbf{R}_{+}$such that $F(t, u, v)$ is nondecreasing in $u$ and $v$ and

$$
|f(x, u, p)| \leq F(|x|,|u|,|p|), \quad x \in \Omega_{\alpha}, u \in \mathbf{R}_{+}, p \in \mathbf{R}^{N} .
$$

$\left(\mathrm{B}_{4}\right)$ For each fixed $(t, u, v) \in[\alpha, \infty) \times \mathbf{R}_{+} \times \mathbf{R}_{+}, \lambda^{-1} F(t, \lambda u, \lambda v)$ is a nondecreasing function of $\lambda \in(0, \infty)$ with $\lim _{\lambda \rightarrow 0+} \lambda^{-1} F(t, \lambda u, \lambda v)=0$ (superlinearity).

A companion theorem will also be proved with $\left(\mathrm{B}_{4}\right)$ replaced by the sublinear hypothesis $\left(\mathrm{B}_{4}^{*}\right)$ below:

$\left(\mathrm{B}_{4}^{*}\right)$ For each fixed $(t, u, v) \in[\alpha, \infty) \times \mathbf{R}_{+} \times \mathbf{R}_{+}, \mu^{-1} F(t, \mu u, \mu v)$ is a nonincreasing function of $\mu \in(0, \infty)$ with $\lim _{\mu \rightarrow \infty} \mu^{-1} F(t, \mu u, \mu v)=0$.

The existence theorems below are proved under one of the following hypotheses:

$$
\begin{aligned}
& \int_{\alpha}^{\infty} p_{2}(t) \frac{z_{2}(t)}{z_{1}(t)} F\left(t, a z_{1}(t), b p(t)\right) d t<\infty, \\
& \int_{\alpha}^{\infty} p_{2}(t) F\left(t, a z_{2}(t), \frac{b p(t) z_{2}(t)}{z_{1}(t)}\right) d t<\infty
\end{aligned}
$$

for some positive constants $a$ and $b$, where $z_{1}(t)$ and $z_{2}(t)$ are positive solutions of (3.1) as described above, and

$$
\begin{aligned}
& p_{2}(t)=\left[z_{1}(t)\left(\frac{z_{2}}{z_{1}}\right)^{\prime}(t)\right]^{-1}, \\
& p(t)=z_{1}(t) \frac{d}{d t} \log \left[\frac{z_{2}(t)}{z_{1}(t)}\right]+\left|z_{1}^{\prime}(t)\right| .
\end{aligned}
$$

These forms of $p_{2}(t)$ and $p(t)$ follow from (2.3) and (2.4).

THEOREM 3.1. If $\left(\mathrm{B}_{1}\right)-\left(\mathrm{B}_{3}\right)$ and either $\left(\mathrm{B}_{4}\right)$ or $\left(\mathrm{B}_{4}^{*}\right)$ hold, then (3.2) is a sufficient condition for equation (1.1) to have infinitely many positive solutions $u(x)$ such that $u(x) / z_{1}(|x|)$ is bounded above and below by positive constants in $\Omega_{\alpha}$.

THEOREM 3.2. If $\left(\mathrm{B}_{1}\right)-\left(\mathrm{B}_{3}\right)$ and either $\left(\mathrm{B}_{4}\right)$ or $\left(\mathrm{B}_{4}^{*}\right)$ hold, then (3.3) is sufficient for equation (1.1) to have infinitely many positive solutions $u(x)$ such that $u(x) / z_{2}(|x|)$ is bounded above and below by positive constants in $\Omega_{\alpha}$. 
THEOREM 3.3. If $\left(\mathrm{B}_{1}\right)-\left(\mathrm{B}_{3}\right)$ and either $\left(\mathrm{B}_{4}\right)$ or $\left(\mathrm{B}_{4}^{*}\right)$ hold, then (3.3) is sufficient for the boundary value problem (1.2) to have infinitely many positive solutions in $\Omega_{\alpha}$.

Proof of Theorem 3.1. Consider the ordinary differential equations

$$
\begin{array}{ll}
L y=F\left(t, y,\left|y^{\prime}\right|\right), & t \geq \alpha, \\
L Y=-F\left(t, Y,\left|Y^{\prime}\right|\right), & t \geq \alpha,
\end{array}
$$

where $L$ is the linear operator given by (3.1). Since (3.4) and (3.5) have the form (2.1), Hypotheses $\left(B_{1}\right),\left(B_{3}\right)$, and $\left(B_{4}\right)$ (respectively, $\left(B_{4}^{*}\right)$ ) show that Theorem 2.1 (respectively, Theorem 2.2) is applicable. By (2.4), $p_{1}=$ $\left(z_{2} / z_{1}\right)^{\prime}$ and $P=z_{2} / z_{1}+$ constant. Then condition (2.5) reduces to (3.2), and it follows from Theorem 2.1 (i) or Theorem 2.2 (i) that equations (3.4) and (3.5) have positive solutions $y(t)$ and $Y(t)$, respectively, in the interval $[\alpha, \infty)$ such that

$$
\lim _{t \rightarrow \infty} \frac{y(t)}{z_{1}(t)}=2 k, \quad \lim _{t \rightarrow \infty} \frac{Y(t)}{z_{1}(t)}=2 K
$$

for all sufficiently small positive constants $k$ and $K$ (or all sufficiently large $k$ and $K$ in the case of hypothesis $\left.\left(\mathrm{B}_{4}^{*}\right)\right)$. These constants can be chosen so that

$$
0<y(t)<Y(t) \text { for all } t \geq \alpha .
$$

Indeed, in the case of hypothesis $\left(\mathrm{B}_{4}\right)$, let $Y(t)$ be determined first satisfying

$$
K \leq \frac{Y(t)}{z_{1}(t)} \leq 3 K, \quad t \geq \alpha,
$$

as in the construction of Theorem 2.1 (i). Then choose $0<k \leq K / 3$ and an associated $y(t)=y(t, k)$ satisfying

$$
k \leq \frac{y(t)}{z_{1}(t)} \leq 3 k \leq K \leq \frac{Y(t)}{z_{1}(t)}, \quad t \geq \alpha .
$$

The argument establishing (3.6) is similar in the case of hypothesis $\left(\mathrm{B}_{4}^{*}\right)$.

We define functions $w$ and $v: \Omega_{\alpha} \rightarrow \mathbf{R}_{+}$by

$$
w(x)=y(|x|), \quad v(x)=Y(|x|), \quad x \in \Omega_{\alpha} .
$$

Then (3.1), (3.4), and (3.5) show that $w$ and $v$ satisfy

$$
\begin{array}{ll}
\Delta w-q(|x|) w=F(|x|, w,|\nabla w|), & x \in \Omega_{\alpha}, \\
\Delta v-q(|x|) v=-F(|x|, v,|\nabla v|), & x \in \Omega_{\alpha},
\end{array}
$$


respectively, and hence by $\left(B_{3}\right)$ they satisfy the elliptic inequalities

$$
\begin{array}{ll}
\Delta w-q(|x|) w \geq f(x, w, \nabla w), & x \in \Omega_{\alpha}, \\
\Delta v-q(|x|) v \leq f(x, v, \nabla v), & x \in \Omega_{\alpha} .
\end{array}
$$

Since $0<w(x)<v(w)$ throughout $\Omega_{\alpha}$ by (3.6), it follows [7, p. 125] that there exists a solution $u(x)$ of

$$
\Delta u-q(|x|) u=f(x, u, \nabla u), \quad x \in \Omega_{\alpha},
$$

with locally Hölder continuous second derivatives in $\Omega_{\alpha}$ such that $w(x) \leq$ $u(x) \leq v(x)$ for all $x \in \Omega_{\alpha}$. As indicated by (3.7), this implies that

$$
k \leq \frac{y(|x|)}{z_{1}(|x|)} \leq \frac{u(x)}{z_{1}(|x|)} \leq \frac{Y(|x|)}{z_{1}(|x|)} \leq 9 k
$$

throughout $\Omega_{\alpha}$.

This completes the proof of Theorem 3.1. The proofs of Theorems 3.2 and 3.3 are virtually the same by application of Theorems 2.1 (ii) and 2.2 (ii).

EXAMPLE 3.4. If $q(t)$ is identically zero, (1.1) reduces to

$$
\Delta u=f(x, u, \nabla u), \quad x \in \Omega_{\alpha} .
$$

In this case a fundamental set of asymptotically ordered positive solutions of $L z=0$ in $[\alpha, \infty)$ is

$$
\begin{array}{lll}
z_{1}(t)=1, & z_{2}(t)=\log (t / \varepsilon) & \text { if } N=2, \\
z_{1}(t)=t^{2-N}, & z_{2}(t)=1 & \text { if } N \geq 3,
\end{array}
$$

for $0<\varepsilon<\alpha$. Condition (3.2) is equivalent to

$$
\begin{array}{ll}
\int_{\alpha}^{\infty} t \log t F\left(t, a, \frac{b}{t \log t}\right) d t<\infty & \text { if } N=2, \\
\int_{\alpha}^{\infty} t^{N-1} F\left(t, a t^{2-N}, b t^{1-N}\right) d t<\infty & \text { if } N \geq 3,
\end{array}
$$

and condition (3.3) is equivalent to

$$
\begin{array}{ll}
\int_{\alpha}^{\infty} t F\left(t, a \log t, \frac{b}{t}\right) d t<\infty & \text { if } N=2, \\
\int_{\alpha}^{\infty} t F\left(t, a, \frac{b}{t}\right) d t<\infty & \text { if } N \geq 3 .
\end{array}
$$

Then (3.13) implies that equation (3.11) has infinitely many positive solutions $u(x)$ in $\Omega_{\alpha}$ for any $\alpha>0$ such that $u(x) / z_{1}(|x|)$ is bounded above and below by positive constants in $\Omega_{\alpha}$; and in particular $u(x) \rightarrow 0$ 
as $|x| \rightarrow \infty$ in dimensions $N \geq 3$. Similarly (3.14) implies the conclusions of Theorems 3.2 and 3.3, and in particular implies the existence of infinitely many solutions of (3.11) in $\Omega_{\alpha}$ which are uniformly bounded above and below by positive constants if $N \geq 3, \alpha>0$.

EXAMPLE 3.5. Consider the equation

$$
\Delta u-\rho^{2}|x|^{2 r} u=\phi(x) u^{\gamma}+\psi(x)|\nabla u|^{\beta}, \quad x \in \Omega_{\alpha},
$$

where $\beta, \gamma, \rho, r$ are constants, $\gamma \geq 0,0 \leq \beta \leq 2, \rho>0,2 r$ is a nonnegative integer, and $\phi, \psi$ are locally Hölder continuous functions in $\Omega_{\alpha}$ for some $\alpha>0$. Equation (3.15) is an example of (1.1) in which $q(t)=\rho^{2} t^{2 r}$ and $f(x, u, p)=\phi(x) u^{\gamma}+\psi(x)|p|^{\beta}$. Define

$$
\phi^{*}(t)=\max _{|x|=t}|\phi(x)|, \quad \psi^{*}(t)=\max _{|x|=t}|\psi(x)|,
$$

$$
F(t, u, v)=\phi^{*}(t) u^{\gamma}+\psi^{*}(t) v^{\beta}, \quad t \geq \alpha, u \in \mathbf{R}_{+}, v \in \mathbf{R}_{+} .
$$

Then $|f(x, u, p)| \leq F(|x|,|u|,|p|)$ for all $x \in \Omega_{\alpha}, u \in \mathbf{R}_{+}, p \in \mathbf{R}^{N}$, and hypothesis $\left(\mathrm{B}_{3}\right)$ holds for this choice of $F$.

In the case of (3.15), equation (3.1) becomes

$$
t^{1-N} \frac{d}{d t}\left(t^{N-1} \frac{d z}{d t}\right)-\rho^{2} t^{2 r} z=0, \quad t \geq \alpha,
$$

which possesses linearly independent solutions with the asymptotic behavior $[9$, p. 285]

$$
z_{1}(t) \sim t^{-\lambda} \exp \left[-\frac{\rho t^{r+1}}{r+1}\right], \quad z_{2}(t) \sim t^{-\lambda} \exp \left[\frac{\rho t^{r+1}}{r+1}\right]
$$

as $t \rightarrow \infty$, where $\lambda=(N+r-1) / 2$. Evidently these solutions are asymptotically ordered at $\infty$, and we assume that $\alpha$ has been selected large enough so that they are positive in $[\alpha, \infty)$. Using (2.4) one easily verifies the asymptotic forms

$$
\begin{array}{ll}
p_{1}(t) \sim 2 \rho t^{r} \exp \left[\frac{2 \rho t^{r+1}}{r+1}\right] & \text { as } t \rightarrow \infty \\
p_{2}(t) \sim \frac{t^{\lambda-r}}{2 \rho} \exp \left[-\frac{\rho t^{r+1}}{r+1}\right] & \text { as } t \rightarrow \infty \\
\frac{p_{2}(t) z_{2}(t)}{z_{1}(t)} \sim \frac{t^{\lambda-r}}{2 \rho} \exp \left[\frac{\rho t^{r+1}}{r+1}\right] & \text { as } t \rightarrow \infty .
\end{array}
$$

We note from (3.16) that hypothesis $\left(\mathrm{B}_{4}\right)$ holds if

$$
\gamma>1 \text { and } \beta>1
$$

and hypothesis $\left(\mathrm{B}_{4}^{*}\right)$ holds if

$$
0 \leq \gamma<1 \text { and } 0 \leq \beta<1 .
$$


In the present example, condition (3.2) is satisfied if both

$$
\int_{\alpha}^{\infty} t^{\lambda(1-\gamma)-r} \exp \left[\frac{\rho(1-\gamma) t^{r+1}}{r+1}\right] \phi^{*}(t) d t<\infty
$$

and

$$
\int_{\alpha}^{\infty} t^{(\lambda-r)(1-\beta)} \exp \left[\frac{\rho(1-\beta) t^{r+1}}{r+1}\right] \psi^{*}(t) d t<\infty,
$$

and condition (3.3) holds if both

$$
\int_{\alpha}^{\infty} t^{\lambda(1-\gamma)-r} \exp \left[\frac{\rho(\gamma-1) t^{r+1}}{r+1}\right] \phi^{*}(t) d t<\infty
$$

and

$$
\int_{\alpha}^{\infty} t^{(\lambda-r)(1-\beta)} \exp \left[\frac{\rho(\beta-1) t^{r+1}}{r+1}\right] \psi^{*}(t) d t<\infty .
$$

We conclude from Theorem 3.1 under hypotheses (3.20), (3.21), and either (3.18) or (3.19) that equation (3.15) has infinitely many bounded positive solutions $u(x)$ in $\Omega_{\alpha}$ such that

$$
c_{1}|x|^{-\lambda} \exp \left[-\frac{\rho|x|^{r+1}}{r+1}\right] \leq u(x) \leq c_{2}|x|^{-\lambda} \exp \left[-\frac{\rho|x|^{r+1}}{r+1}\right],
$$

$x \in \Omega_{\alpha}$, for some positive constants $c_{1}$ and $c_{2}$. Furthermore, Theorem 3.2 shows that (3.22), (3.23), and either (3.18) or (3.19) imply the existence of infinitely many unbounded positive solutions $u(x)$ of (3.15) in $\Omega_{\alpha}$ such that

$$
c_{3}|x|^{-\lambda} \exp \left[\frac{\rho|x|^{r+1}}{r+1}\right] \leq u(x) \leq c_{4}|x|^{-\lambda} \exp \left[\frac{\rho|x|^{r+1}}{r+1}\right], \quad x \in \Omega_{\alpha},
$$

for some positive constants $c_{3}$ and $c_{4}$. Theorem 3.3 shows that the same conditions are sufficient for the existence of positive solutions of (3.15) in $\Omega_{\alpha}$ satisfying the boundary condition $\left.u\right|_{\partial \Omega_{\alpha}}=0$.

Example 3.5 can be extended to mixed sublinear-superlinear equations (3.15), in which $\gamma>1$ and $0 \leq \beta<1$, via the corollary below. More generally we consider equations (1.1) with the structure

$$
\Delta u-q(|x|) u=f_{1}(x, u, \nabla u)-f_{2}(x, u, \nabla u), \quad x \in \Omega_{\alpha},
$$

where each $f_{i}$ satisfies hypotheses $\left(\mathrm{B}_{1}\right),\left(\mathrm{B}_{2}\right), f_{1}$ satisfies $\left(\mathrm{B}_{4}\right),\left(f_{2}\right)$ satisfies $\left(B_{4}^{*}\right)$, but $\left(B_{3}\right)$ is replaced by $\left(B_{3}^{*}\right)$ below:

$\left(\mathrm{B}_{3}^{*}\right)$ There exist continuous functions $F_{i}: \mathbf{R}_{+}^{3} \rightarrow \mathbf{R}_{+}, i=1,2$, such that each $F_{i}(t, u, v)$ is nondecreasing in $u$ and $v$ and

$$
\begin{aligned}
& 0 \leq f_{1}(x, u, p) \leq F_{1}(|x|, u,|p|) \\
& 0 \leq-f_{2}(x, u, p) \leq F_{2}(|x|, u,|p|)
\end{aligned}
$$

for all $x \in \Omega_{\alpha}, u \in \mathbf{R}_{+}, p \in \mathbf{R}^{N}$. 
Corollary 3.6. Suppose that $q$ and each $f_{i}$ satisfies $\left(\mathrm{B}_{1}\right),\left(\mathrm{B}_{2}\right),\left(\mathrm{B}_{3}^{*}\right)$, and $(3.2)$, and that $f_{1}, f_{2}$ satisfy $\left(\mathrm{B}_{4}\right),\left(\mathrm{B}_{4}^{*}\right)$, respectively, with $F$ replaced by $F_{1}$, $F_{2}$, respectively. Then equation (3.24) has infinitely many positive solutions $u(x)$ such that $u(x) / z_{1}(|x|)$ is bounded above and below by positive constants throughout $\Omega_{\alpha}$.

Proof. Consider the elliptic equations

$$
\begin{aligned}
& \Delta w-q(|x|) w=f_{1}(x, w, \nabla w) \\
& \Delta v-q(|x|) v=-f_{2}(x, v, \nabla v)
\end{aligned}
$$

in $\Omega_{\alpha}$. By Theorem 3.1, these equations have solutions $v, w \in C_{\mathrm{loc}}^{2+\lambda}\left(\Omega_{\alpha}\right)$ such that

$$
\begin{array}{ll}
k_{1} z_{1}(|x|) \leq w(x) \leq k_{2} z_{1}(|x|), & x \in \Omega_{\alpha}, \\
k_{3} z_{1}(|x|) \leq v(x) \leq k_{4} z_{1}(|x|), & x \in \Omega_{\alpha},
\end{array}
$$

for all sufficiently small positive constants $k_{1}, k_{2}>k_{1}$ and sufficiently large positive constants $k_{3}, k_{4}>k_{3}$. Since each $f_{i} \geq 0, w$ and $v$ satisfy the elliptic differential inequalities

$$
\begin{aligned}
& \Delta w-q(|x|) w \geq f_{1}(x, w, \nabla w)-f_{2}(x, w, \nabla w), \\
& \Delta v-q(|x|) v \leq f_{1}(x, v, \nabla v)-f_{2}(x, v, \nabla v),
\end{aligned}
$$

respectively, in $\Omega_{\alpha}$. We can choose $k_{3}$ large enough so that $0<w(x) \leq$ $v(x)$ throughout $\Omega_{\alpha}$, and it follows [7, p. 125] as in Theorem 3.1 that (3.24) has a solution $u \in C_{\mathrm{loc}}^{2+\lambda}\left(\Omega_{\alpha}\right)$ satisfying $w(x) \leq u(x) \leq v(x)$ throughout $\Omega_{\alpha}$.

Corollary 3.7. Let $q$ and each $f_{i}$ be as in Corollary 3.6 except that (3.2) is replaced by (3.3). Then equation (3.24) has infinitely many positive solutions $u(x)$ such that $u(x) / z_{2}(|x|)$ is bounded above and below by positive constants throughout $\Omega_{\alpha}$.

The proof, based on Theorem 3.2, is similar to that of Corollary 3.6.

These corollaries give new global existence theorems even for semilinear equations of the type

$$
\Delta u=\phi(x) u^{\gamma}-\psi(x) u^{\beta}, \quad x \in \Omega_{\alpha},
$$

where $\gamma>1,0 \leq \beta<1$, and $\phi$ and $\psi$ are nonnegative locally Hölder continuous functions in $\Omega_{\alpha}, \alpha>0$. Corollaries 3.6 and 3.7 and Example 3.4 imply the following additional corollaries. 
COROLlARY 3.8. Equation (3.26) has a positive solution which is bounded and bounded away from zero in an arbitrary exterior domain $\Omega \subset \mathbf{R}^{2}$ if both

$$
\int_{\alpha}^{\infty} t \log t \phi^{*}(t) d t<\infty \text { and } \int_{\alpha}^{\infty} t \log t \psi^{*}(t) d t<\infty
$$

for some $\alpha>0$.

For example, we can choose

$$
\alpha=\inf \{|x|: x \in \partial \Omega\}
$$

to obtain $\Omega \subset \Omega_{\alpha}$, and apply the earlier results to $\Omega_{\alpha}$.

COROLlaRy 3.9. Equation (3.26) has a positive solution which is bounded above and below by positive constant multiples of $\log (|x| / \varepsilon)$ in an arbitrary domain $\Omega_{\alpha} \subset \mathbf{R}^{2}, 0<\varepsilon<\alpha$, if both

$$
\int^{\infty} t(\log t)^{\gamma} \phi^{*}(t) d t<\infty \text { and } \int^{\infty} t(\log t)^{\beta} \psi^{*}(t) d t<\infty
$$

COROLlaRY 3.10. Equation (3.26) has a positive solution which is bounded and bounded away from zero in an arbitrary exterior domain $\Omega \subset \mathbf{R}^{N}, N \geq 3$, if both

$$
\int^{\infty} t \phi^{*}(t) d t<\infty \text { and } \int^{\infty} t \psi^{*}(t) d t<\infty
$$

COROllary 3.11. Equation (3.26) has a positive solution which is bounded above and below by positive constant multiples of $|x|^{2-N}$ in an arbitrary exterior domain $\Omega \subset \mathbf{R}^{N}, N \geq 3$, if both

$$
\int^{\infty} t^{\sigma} \phi^{*}(t) d t<\infty \text { and } \int^{\infty} t^{\rho} \psi^{*}(t) d t<\infty,
$$

where $\sigma=(N-1)-\gamma(N-2), \rho=(N-1)-\beta(N-2)$.

Theorems 3.1-3.3 also apply to quasilinear equations of the type

$$
\Delta u-q(|x|) u=\phi(x) u^{\gamma}+u^{\beta} \sum_{i, j=1}^{N} \psi_{i j}(x) \frac{\partial u}{\partial x_{i}} \frac{\partial u}{\partial x_{j}},
$$

where $\phi$ and each $\psi_{i j}$ are locally Hölder continuous in $\Omega_{\alpha}$, and the matrix $\left(\psi_{i j}(x)\right)$ is symmetric and positive semidefinite in $\Omega_{\alpha}$. 


\section{REFERENCES}

[1] N. Kawano, On bounded entire solutions of semilinear elliptic equations, Hiroshima Math. J., 14 (1984), 125-158.

[2] N. Kawano and M. Naito, Positive solutions of semilinear second order elliptic equations in exterior domains, Hiroshima Math. J., 12 (1982), 141-149.

[3] C. E. Kenig and W-M. Ni, An exterior Dirichlet problem with applications to some nonlinear equations arising in geometry, Amer. J. Math., 106 (1984), 689-702.

[4] Takaŝi Kusano and Shinnosuke Oharu, Bounded entire solutions of second order semilinear elliptic equations with application to a parabolic initial value problem, Indiana Univ. Math. J., 34 (1985), 85-95.

[5] W-M. Ni, On the elliptic equation $\Delta u+K(x) u^{(N+2) \wedge(N-2)}=0$, its generalizations, and applications in geometry, Indiana Univ. Math. J., 31 (1982), 493-529.

[6] E. S. Noussair and C. A. Swanson, Positive solutions of semilinear Schrödinger equations in exterior domains, Indiana Univ. Math. J., 28 (1979), 993-1003.

[7] Positive solutions of quasilinear elliptic equations in exterior domains, J. Math. Anal. Appl., 75 (1980), 121-133.

[8] _ Global positive solutions of semilinear elliptic equations, Canad. J. Math., 35 (1983), 839-861.

[9] L. Sirovich, Techniques of Asymptotic Analysis, Springer-Verlag, New York-Heidelberg-Berlin, 1971.

[10] C. A. Swanson, Criteria for oscillatory sublinear Schrödinger equations, Pacific J. Math., 104 (1983), 483-493.

[11] Extremal positive solutions of semilinear Schrödinger equations, Canad. Math. Bull., 26 (1983), 171-178.

[12] W. F. Trench, Canonical forms and principal systems for general disconjugate equations, Trans. Amer. Math. Soc., 189 (1974), 319-327.

Received February 24, 1984. The first author was supported in part by Grant-in-Aid for Scientific Research (No. 58460004), Ministry of Education (Japan). The second author gratefully acknowledges support from NSERC (Canada) under Grant A-3105.

HIROSHIMA UNIVERSITY

HiRoSHIMA, JAPAN 730

AND

UNIVERSITY OF BRITISH COLUMBIA

VANCOUVER, B. C.

CANADA V6T 1 Y4 



\section{PACIFIC JOURNAL OF MATHEMATICS EDITORS}

\author{
V. S. VARADARAJAN (Managing Editor) \\ University of California \\ Los Angeles, CA 90024 \\ Charles R. DePrima \\ California Institute of Technology \\ Pasadena, CA 91125 \\ R. FINN \\ Stanford University \\ Stanford, CA 94305
}

\author{
HeRmanN FlaschKa \\ University of Arizona \\ Tucson, AZ 857.21
}

RAMESH A. GANGOLli

University of Washington

Seattle, WA 98195

ROBION KIRBY

University of California

Berkeley, CA 94720
C. C. MOORE

University of California

Berkeley, CA 94720

H. SAMELSON

Stanford University

Stanford, CA 94305

HAROLD STARK

University of California, San Diego

La Jolla, CA 92093

\section{ASSOCIATE EDITORS}
R. ARENS
E. F. BECKENBACH
B. H. NeumanN
F. WOLF
K. YoSHIDA
(1906-1982)

\section{SUPPORTING INSTITUTIONS}

\begin{abstract}
UNIVERSITY OF ARIZONA
UNIVERSITY OF BRITISH COLUMBIA

CALIFORNIA INSTITUTE OF TECHNOLOGY

UNIVERSITY OF CALIFORNIA

MONTANA STATE UNIVERSITY

UNIVERSITY OF NEVADA, RENO

NEW MEXICO STATE UNIVERSITY

OREGON STATE UNIVERSITY
\end{abstract}

\author{
UNIVERSITY OF OREGON \\ UNIVERSITY OF SOUTHERN CALIFORNIA \\ STANFORD UNIVERSITY \\ UNIVERSITY OF HAWAII \\ UNIVERSITY OF TOKYO \\ UNIVERSITY OF UTAH \\ WASHINGTON STATE UNIVERSITY \\ UNIVERSITY OF WASHINGTON
}

The Supporting Institutions listed above contribute to the cost of publication of this Journal, but they are not owners or publishers and have no responsibility for its content or policies.

Mathematical papers intended for publication in the Pacific Journal of Mathematics should be in typed form or offset-reproduced (not dittoed), double spaced with large margins. Please do not use built up fractions in the text of the manuscript. However, you may use them in the displayed equations. Underline Greek letters in red, German in green, and script in blue. The first paragraph must be capable of being used separately as a synopsis of the entire paper. In particular it should contain no bibliographic references. Please propose a heading for the odd numbered pages of less than 35 characters. Manuscripts, in triplicate, may be sent to any one of the editors. Please classify according to the scheme of Math. Reviews, Index to Vol. 39. Supply name and address of author to whom proofs should be sent. All other communications should be addressed to the managing editor, or Elaine Barth, University of California, Los Angeles, California 90024.

There are page-charges associated with articles appearing in the Pacific Journal of Mathematics. These charges are expected to be paid by the author's University, Government Agency or Company. If the author or authors do not have access to such Institutional support these charges are waived. Single authors will receive 50 free reprints; joint authors will receive a total of 100 free reprints. Additional copies may be obtained at cost in multiples of 50 .

The Pacific Journal of Mathematics is issued monthly as of January 1966. Regular subscription rate: $\$ 190.00$ a year (5 Vols., 10 issues). Special rate: $\$ 66.00$ a year to individual members of supporting institutions.

Subscriptions, orders for numbers issued in the last three calendar years, and changes of address should be sent to Pacific Journal of Mathematics, P.O. Box 969, Carmel Valley, CA 93924, U.S.A. Old back numbers obtainable from Kraus Periodicals Co., Route 100, Millwood, NY 10546.

The Pacific Journal of Mathematics at P.O. Box 969, Carmel Valley, CA 93924 (ISSN 0030-8730) publishes 5 volumes per year. Application to mail at Second-class postage rates is pending at Carmel Valley, California, and additional mailing offices. Postmaster: Send address changes to Pacific Journal of Mathematics, P.O. Box 969, Carmel Valley, CA 93924.

PUBLISHED BY PACIFIC JOURNAL OF MATHEMATICS, A NON-PROFIT CORPORATION

Copyright $\odot 1985$ by Pacific Journal of Mathematics 


\section{Pacific Journal of Mathematics}

Vol. 120, No. $2 \quad$ October, 1985

Philip Marshall Anselone and Mike Treuden, Regular operator

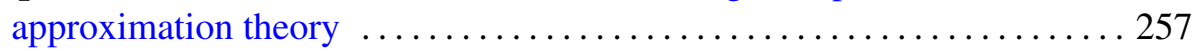

Giuseppe Baccella, Semiprime $\aleph-Q F 3$ rings $\ldots \ldots \ldots \ldots \ldots \ldots \ldots \ldots . \ldots \ldots$

Earl Robert Berkson and Thomas Alastair Gillespie, The generalized M.

Riesz theorem and transference $\ldots \ldots \ldots \ldots \ldots \ldots \ldots \ldots . \ldots 279$

Joachim Boidol, A Galois-correspondence for general locally compact

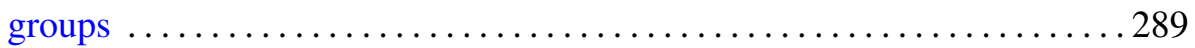

Joseph Eugene D'Atri, Josef Dorfmeister and Yan Da Zhao, The isotropy

representation for homogeneous Siegel domains ............... 295

C. Debiève, On Banach spaces having a Radon-Nikodým dual

Michael Aaron Freedman, Existence of strong solutions to singular

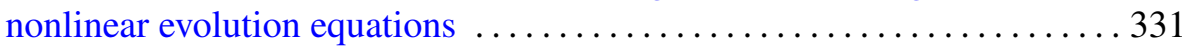

Francisco Jose Freniche, Grothendieck locally convex spaces of continuous

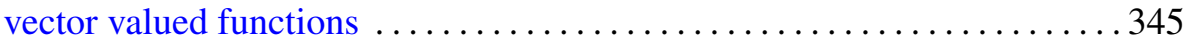

Hans-Peter Künzi and Peter Fletcher, Extension properties induced by complete quasi-uniformities . ............................ 357

Takaŝi Kusano, Charles Andrew Swanson and Hiroyuki Usami, Pairs of

positive solutions of quasilinear elliptic equations in exterior domains . . 385

Angel Rafael Larotonda and Ignacio Zalduendo, Spectral sets as Banach

manifolds

J. Martínez-Maurica and C. Pérez García, A new approach to the

Kreı̆n-Milman theorem

Christian Pommerenke, On the boundary continuity of conformal maps . . . 423

M. V. Subba Rao, Some Rogers-Ramanujan type partition theorems

Stephen Edwin Wilson, Bicontactual regular maps .........

Jaap C. S. P. van der Woude, Characterizations of (H)PI extensions

Kichoon Yang, Deformation of submanifolds of real projective space

Subhashis Nag, Errata: "On the holomorphy of maps from a complex to a

real manifold" 